\title{
Adiabatic Thermal Radiation Pumps for Thermal Photonics
}

\author{
Huanan Li $^{1,2 *}$, Lucas J. Fernández-Alcázar ${ }^{1 *}$, Fred Ellis ${ }^{1}$, Boris Shapiro ${ }^{3}$, Tsampikos Kottos ${ }^{1}$ \\ ${ }^{1}$ Wave Transport in Complex Systems Lab, Department of Physics, \\ Wesleyan University, Middletown, CT-06459, USA \\ ${ }^{2}$ Photonics Initiative, Advanced Science Research Center, CUNY, NY 10031, USA \\ ${ }^{3}$ Technion - Israel Institute of Technology, Technion City, Haifa 32000, Israel
}

(Dated: June 4, 2019)

\begin{abstract}
We control the direction and magnitude of thermal radiation, between two bodies at equal temperature (in thermal equilibrium), by invoking the concept of adiabatic pumping. Specifically, within a resonant near-field electromagnetic heat transfer framework, we utilize an instantaneous scattering matrix approach to unveil the critical role of wave interference in radiative heat transfer. We find that appropriately designed adiabatic pumping cycling near diabolic singularities can dramatically enhance the efficiency of the directional energy transfer. We confirm our results using a realistic electronic circuit set-up.
\end{abstract}

Introduction - Understanding the rules that dictate thermal radiation and the development of novel schemes that allow us to tame its flow, has offered over the years an exciting arena of research [1-4]. From one side, there are fundamental challenges associated with basic constraints that need to be understood in order to manage thermal radiation [4-8]. At their core are questions associated with the importance of thermal electromagnetic fluctuations and their implications in directional thermal radiation. On the other hand, there is a wide range of applications that can benefit from advances in thermal radiation management. In fact, in close connection with the rapid developments that we are witnessing in the field of nanophotonics, the subfield of thermal photonics has emerged [9-20] and promises to revolutionize modern energy technologies. Examples include thermophotovoltaics [21-24], thermal imaging [25, 26], thermal circuits [27-29], and radiative cooling [30].

In this paper we propose to manipulate thermal radiation by introducing the concept of an adiabatic thermal radiation pump. The pump operates between two reservoirs that are maintained at the same temperature - as opposed to common approach where heat flow requires a temperature gradient. A possible setup of a thermal radiation pump is depicted in Fig. 1a. By slow periodic modulation of the eigenfrequencies of two resonators, coupled to two separate reservoirs at equal temperature $T$, one can transfer thermal radiation from one reservoir to the other (see green arrows). This process of creating a directional radiation flow may be termed adiabatic thermal radiation pumping. The amount of heat pumped in one cycle depends on the details of the modulation process. In particular, we show that the existence of a diabolic point (i.e. an exact degeneracy) in the spectrum of the system's Hamiltonian leads to a dramatic enhancement of the effect, for an appropriately chosen modulation cy-

[*]These authors contributed equally to the results of this paper
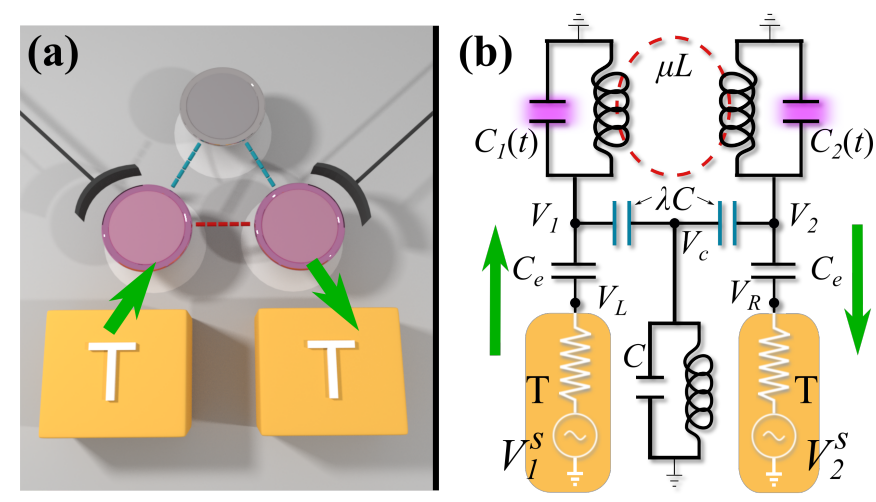

FIG. 1: Proposed implementations of our thermal radiation pumping scheme: (a) A nano-photonic structure consisting of three single-mode resonators. The resonant frequencies of the first and third resonator (purple colors) are periodically modulated via a weak adiabatic modulation of the permittivities of the resonators. The system is in contact with two independent baths at the same temperature $T$. (b) A circuit consisting of three LC resonators. Two of these resonators are modulated via their (purple) capacitances. The circuit is coupled capacitively to two artificial reservoirs at the same temperature $T$. The reservoirs are implemented by synthesized noise sources generating random voltages $V_{1,2}^{s}$ with a prescribed spectral distribution. The positive direction for the pumped flow is chosen to match the green arrows.

cle. Our theoretical results are based on a coupled-modetheory (CMT) approach to resonant thermal radiation and are backed up by detailed numerical simulations using realistic circuit set-ups, see Fig. 1b. Our approach unveils the importance of wave interference in radiative heat transfer by connecting the pumped thermal radiative current with the instantaneous reflection phase. This connection opens up new possibilities in the field of thermal photonics. Our concept of the adiabatic thermal radiation pumping is inspired by adiabatic charge pumping in condensed matter, where a DC current in response to a slowly varying time-periodic potential has been proposed [31-36] and experimentally demonstrated [37]. 
CMT Modeling of Thermal Radiation- We consider a photonic circuit supporting a finite number of resonant modes $N_{s}$ described by a Hermitian Hamiltonian $H_{0}$. The system is in contact with two separate heat baths at constant temerature (see Fig. 1a where $N_{s}=3$ ) via leads with the system-lead coupling described by an operator $\hat{W}$.

At thermal equilibrium, the two baths generate photons at frequency $\omega$ with mean number $\Theta_{T}(\omega)=$ $\left(e^{\frac{\hbar \omega}{k_{B} T}}-1\right)^{-1}$ given by the Bose-Einstein statistics. The radiative thermal energy exchange between the two heat baths can be studied using a time-dependent CMT $[38,39]$

$$
\begin{aligned}
\imath \frac{d}{d t} \Psi & =H_{\mathrm{eff}} \Psi+\imath \hat{W} \theta^{(+)} ; \quad H_{\mathrm{eff}}=\left(H_{0}+\Lambda-\frac{\imath}{2} \hat{W} \hat{W}^{T}\right) \\
\theta^{(-)} & =\hat{W}^{T} \Psi-\theta^{(+)}
\end{aligned}
$$

where $\Psi=\left(\psi_{1}, \psi_{2}, \cdots, \psi_{N_{s}}\right)^{T}$ describes the modal amplitude of the field and it is normalized in a way that $\left|\psi_{s}\right|^{2}$ represents the energy of the $s^{t h}$ mode. The variables $\theta_{n}^{( \pm)}(\omega)$ (frequency domain of $\theta^{ \pm}$in Eq. (1)) indicate the flux-amplitudes of the incoming $(+)$ and outgoing (-) waves from and towards the reservoir $n=1,2$ via leads. At thermal equilibrium the incoming flux (from the reservoirs) satisfies the correlation relation [38]

$$
\left\langle\theta_{n}^{(+)}(\omega)\left(\theta_{m}^{(+)}\left(\omega^{\prime}\right)\right)^{*}\right\rangle=\frac{\hbar \omega}{2 \pi} \Theta_{T}(\omega) \delta\left(\omega-\omega^{\prime}\right) \delta_{n m}
$$

and, therefore, the outgoing power from the $n^{\text {th }}$ heat bath is given by the double integral over frequency $\omega$ of this correlation function.

The associated scattering matrix $S$, connecting the outgoing $\theta^{-}(\omega)$ to the incoming $\theta^{+}(\omega)$ waves, can be evaluated using Eq. (1). We have [40]

$$
S=-I_{2}-\imath W^{T} G_{\mathrm{eff}} W, \quad G_{\mathrm{eff}}=\frac{1}{H_{\mathrm{eff}}-\omega I_{N_{s}}}
$$

where $I_{n}$ is the $n \times n$ identity matrix. The matrix $W$, describes the coupling to the leads (in frequency domain), and has dimensions $N_{s} \times 2$. Its elements are $W_{s, n}=\sqrt{v_{g}} w_{n} \delta_{s n}$ where $w_{n}$ are dimensionless coupling strengths, and $v_{g}=\frac{\partial \omega(k)}{\partial k}$. Finally, the matrix $\Lambda$ appearing in $H_{\text {eff }}$ (see Eq. (1)) is a renormalization term due to the coupling of the system with the leads and it is specific to the properties of the leads.

Thermal Radiation Pumps- Next, we consider a system whose Hamiltonian $H_{0}\left(u^{t}, v^{t}\right)$ depends on two timevarying independent parameters $\left(u^{t}, v^{t}\right)$. We further assume that these parameters are periodically modulated in time with frequency $\Omega$, such that $H_{0}(t)=$ $H_{0}(t+2 \pi / \Omega)$. During one period of the modulation these parameters form a closed cycle in the $\left(u^{t}, v^{t}\right)$ parameter space. The associated enclosed "pumping area" is $\mathcal{A} \equiv \int_{0}^{2 \pi / \Omega} d t u^{t} \frac{d v^{t}}{d t}$. We want to analyze the net radiative energy flux from one bath to another during one pumping circle.

We consider circumstances where the variation of $\left(u^{t}, v^{t}\right)$ is small such that $\mathcal{A} \rightarrow 0$. We want to evaluate the total radiative (time-averaged) thermal energy flux per pumping area $\overline{\mathcal{I}}$. The latter is:

$\overline{\mathcal{I}} \equiv \frac{\Omega}{2 \pi} \int \frac{d \omega}{2 \pi} \hbar \omega \Theta_{T}(\omega) Q(\omega), Q(\omega) \equiv \lim _{\mathcal{A} \rightarrow 0} \frac{\int_{0}^{\frac{2 \pi}{\Omega}} d t \mathcal{I}_{x_{0}}(t, \omega)}{\mathcal{A}}$

where $Q(\omega)$ is the radiative energy density (i.e. per area in the parameter space) and $\mathcal{I}_{x_{0}}(t, \omega)$ is the dimensionless (normalized) time-dependent directional net energy current, at some observation cross section at $x=x_{0}$ within the leads. The latter is evaluated under the condition of two uncorrelated counter propagating incoming waves of frequency $\omega$ and unit flux. In the case where $H_{0}$ is static, the net thermal radiative current $\mathcal{I}$ at each lead is zero. From Eq. (4) it is clear that an understanding of $Q(\omega)$ is essential for the analysis and control of $\overline{\mathcal{I}}$ [41].

Adiabatic Pumping- In the adiabatic limit, $\Omega \rightarrow 0$, the study of radiative thermal energy $Q(\omega)$ boils down to the analysis of the instantaneous scattering matrix $S^{t}$ [31]. The latter is given in terms of Eq. (3) with the superscript $t$ indicating the parametric dependence of the matrix elements of $S$ at a specific instant $t$ during the pumping cycle. It can be generally parametrized in terms of three independent parameters: the instantaneous reflectance $R^{t}$, and the instantaneous reflection and transmission phases $\alpha^{t}, \varphi^{t} \in \mathcal{R}$ respectively. Specifically we have:

$$
S^{t}=e^{\imath \varphi^{t}}\left[\begin{array}{cc}
\sqrt{R^{t}} e^{\imath \alpha^{t}} & \imath \sqrt{1-R^{t}} \\
\imath \sqrt{1-R^{t}} & \sqrt{R^{t}} e^{-\imath \alpha^{t}}
\end{array}\right], \quad 0 \leq R^{t} \leq 1 .
$$

Using this parametrization, we write $Q(\omega)$ as

$$
Q(\omega)=\lim _{\mathcal{A} \rightarrow 0} \frac{1}{\mathcal{A}} \frac{\partial}{\partial \omega} \int_{0}^{2 \pi / \Omega} d t R^{t} \frac{d \alpha^{t}}{d t}=\frac{\partial}{\partial \omega}\left|\frac{\partial\left(R^{t}, \alpha^{t}\right)}{\partial\left(u^{t}, v^{t}\right)}\right|
$$

which applies whenever the period of the driving is larger than the delay time that the "photons" dwell inside the scatterer. It is important to stress that Eq. (6) allows us to connect wave interference phenomena (imprinted via the reflection phase $\alpha^{t}$ ) with the thermal radiation problem. Furthermore, it opens up new directions in the field of thermal photonics. Using Eqs. $(5,3)$ we have that

$$
R^{t}=\left|S_{11}^{t}\right|^{2}, \frac{d \alpha^{t}}{d t}=\frac{1}{2 \imath} \frac{d}{d t}\left(\ln \frac{S_{11}^{t}}{S_{22}^{t}}\right),
$$

where the subscripts indicate the matrix elements of $S^{t}$.

Direct inspection of Eqs. $(5,7)$ indicates that the pumped thermal radiation energy will be affected by the proximity of resonant modes where $R$ and $\alpha$ experience 
an abrupt change in $\omega$. One would expect that higher order spectral singularities, like diabolic points (DP), could lead to a dramatic enhancement of $Q(\omega)$. We will show that their effect in $\overline{\mathcal{I}}$ is controlled by the position of the adiabatic cycle in the parameter space, with respect to such spectral singularities.

A prototype CMT model with DPs- We consider a prototype system of three coupled resonant modes, that can support a DP degeneracy. The system is described by the CMT Hamiltonian

$$
H_{0}\left(t ; u^{t}, v^{t}\right)=\left[\begin{array}{ccc}
\omega_{0}+v^{t} & -1 & 1 \\
-1 & \omega_{0} & -1 \\
1 & -1 & \omega_{0}+u^{t}
\end{array}\right]
$$

where $u^{t}=u^{(0)}+\delta u^{t}, v^{t}=v^{(0)}+\delta v^{t}$ with $\left(u^{(0)}, v^{(0)}\right)=$ $(d \cos \theta, d \sin \theta)$ and $\delta u^{t}=\delta u^{t+2 \pi / \Omega}, \delta v^{t}=\delta v^{t+2 \pi / \Omega}$. The pair $\left(u^{(0)}, v^{(0)}\right)$ determines the center of the adiabatic cycle, reparameterized with $d$ and $\theta$ in polar form.

When $u^{t}=v^{t}=0$, the eigen-frequencies of Hamiltonian Eq. (8) are $a_{1}^{(0)}=a_{2}^{(0)}=\omega_{\mathrm{DP}}=\omega_{0}-1$ (DP degeneracy) and $a_{3}^{(0)}=\omega_{0}+2$. This DP degeneracy can be lifted in two ways: (a) by introducing $\left(u^{(0)}, v^{(0)}\right) \neq(0,0)$; (b) by coupling the system to the leads. In the latter case, the eigen-modes turn into resonant modes. The resonant frequencies are the real parts of the poles of the $S$-matrix which are identified with the complex eigenvalues $\left\{a_{n}\right\}$ of the effective Hamiltonian $H_{\text {eff }}^{(0)}$, see Eqs. $(1,3)$ in the absence of modulation. Their corresponding imaginary part is the resonance line-width which describes the decay rate of these modes to the reservoirs. In other words the degenerate eigenmodes $a_{1,2}^{(0)}$ move apart from one-another and turn to $a_{1} \neq a_{2}$ whenever the above two mechanisms are in effect. The degree of repulsion between $\left(a_{1}, a_{2}\right)$ is controlled by the interplay of the proximity of the adiabatic cycle to the DP and the coupling with the leads.

Effects of DP in Pumped Thermal Radiation- In the presence of small time-periodic variations $\left(\delta v^{t}, \delta u^{t}\right)$, the Green's function $G_{\text {eff }}=\left(H_{\text {eff }}-\omega I_{N_{s}}\right)^{-1}$ which appears in the evaluation of $S^{t}$ (and therefore in $Q(\omega)$ ), involves the instantaneous effective Hamiltonian $H_{\text {eff }}=H_{\text {eff }}^{(0)}+\Delta^{t}$ where $\Delta^{t}=\operatorname{diag}\left(\delta v^{t}, 0, \delta u^{t}\right)$. We expand $G_{\text {eff }}$ in a series keeping terms up to first order in $\Delta^{t}$ i.e. $G_{\text {eff }}=G_{\text {eff }}^{(0)}-$ $G_{\text {eff }}^{(0)} \Delta^{t} G_{\text {eff }}^{(0)}+\cdots$ where $G_{\text {eff }}^{(0)}=\left(H_{\text {eff }}^{(0)}-\omega I_{N_{s}}\right)^{-1}$. We can make further progress by representing $G_{\text {eff }}$ in the biorthogonal basis $\left\{\left(a_{i}|,| a_{i}\right)\right\}(i=1,2,3)$ of $H_{\text {eff }}^{(0)}$ where $G_{\text {eff }}^{(0)}$ is diagonal. The reflection coefficient $R^{t}$ and phase $\alpha^{t}$ are evaluated (up to first order in $\delta u^{t}, \delta v^{t}$ ) by direct substitution of $G_{\text {eff }}$ into Eq. (3). Finally, the adiabatic thermal radiation energy $Q$ is calculated using Eq. (6). Specifically, using Eqs. $(5,6,3,7)$, we find that

$$
Q(\omega) \equiv \frac{\partial}{\partial \omega} P\left(\omega, d,\left\{w_{n}\right\}\right)=\lim _{\mathcal{A} \rightarrow 0} \frac{\imath}{\mathcal{A}} \frac{\partial}{\partial \omega}\left\{\int_{0}^{2 \pi / \Omega} d t \operatorname{Re}\left[\left(1+\operatorname{Tr} A_{11}\right) \operatorname{Tr}\left(\tilde{\triangle}^{t} A_{11}\right)^{*}\right] \frac{d \operatorname{Tr}\left(\frac{\tilde{\triangle}^{t} A_{22}}{1+\operatorname{Tr} A_{22}}-\frac{\tilde{\triangle}^{t} A_{11}}{1+\operatorname{Tr} A_{11}}\right)}{d t}\right\}
$$

where $A^{i j}=\imath \frac{\left.W^{T} \mid a_{i}\right)\left(a_{j} \mid W\right.}{\sqrt{a_{i}-\omega} \sqrt{a_{j}-\omega}}$ and $\tilde{\triangle}_{i j}^{t}=\frac{\left(a_{i}\left|\triangle^{t}\right| a_{j}\right)}{\sqrt{a_{i}-\omega} \sqrt{a_{j}-\omega}}$. The implicit subscript (not shown) of matrix $A^{i j}$ denotes the entry while the trace operation is with respect to the dummy variable, e.g., $\operatorname{Tr}\left(\tilde{\triangle}^{t} A_{11}\right)=\sum_{i, j} \tilde{\triangle}_{i j}^{t} A_{11}^{j i}$.

Direct substitution of Eq. (9) in Eq. (4) allows us to perform an integration by parts and express the thermal energy flux per pumping area $\overline{\mathcal{I}}$ as

$$
\overline{\mathcal{I}}=-\frac{\Omega}{2 \pi} \int \frac{d \omega}{2 \pi} \frac{\partial\left[\hbar \omega \Theta_{T}(\omega)\right]}{\partial \omega} P\left(\omega, d,\left\{w_{n}\right\}\right)
$$

where for near-resonant thermal radiation the boundary contributions (associated with the integration by part) are neglected. Using the residue theorem we get

$$
\overline{\mathcal{I}} \propto \sum_{n} \operatorname{Res}\left[\frac{\partial f(\omega)}{\partial \omega} P(\omega) ; \omega_{n}\right] ; \quad \mathcal{I} m\left(\omega_{n}\right)>0
$$

where $f(\omega) \equiv \hbar \omega \Theta(\omega, T)$ and $\omega_{n}$ are the poles of $P(\omega)$ (note that $f(\omega)$ is an analytic function). In the case of near-field resonant thermal transport, these poles can be associated with the poles of the scattering matrix Eq. (3). They originate from the DP degeneracies of the isolated system once they move to the complex frequency plane due to coupling with the leads.

In fact, the singular behavior of $R^{t}$ and $d \alpha^{t} / d t$ will be significant in the presence of a diabolic point degeneracy. In this case a further progress can be made for the evaluation of $\overline{\mathcal{I}}$. Specifically,

$$
\overline{\mathcal{I}} \propto \sum_{n}^{\prime} \operatorname{Res}\left[P(\omega) ; \omega_{n}\right]
$$

where the summation is restricted to poles near the DP.

Examples of Adiabatic Pumps - The above theoretical considerations can be directly tested using the CMT system of Eq. (8). We chose $\theta=45^{0}, \delta u^{t}=r \cos \Omega t$ and $\delta v^{t}=r \sin \Omega t$. Furthermore we assume (left and right) tight-binding leads with dispersion $\omega=\omega_{0}-2 \cos k$ that are coupled to the scattering target with the same cou- 

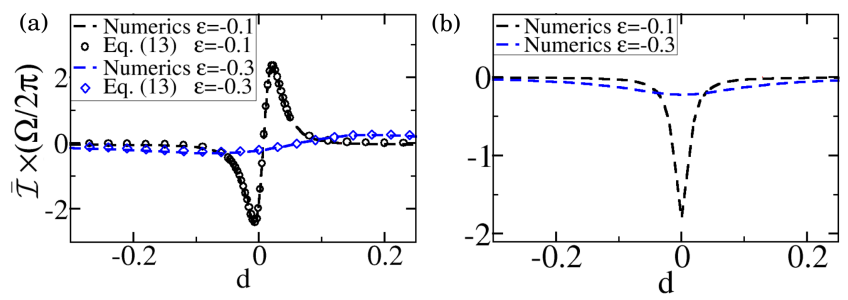

FIG. 2: Numerical evaluation (dashed lines) of rescaled total radiative (time-averaged) thermal energy flux $\overline{\mathcal{I}} \times \frac{2 \pi}{\Omega}$ versus the control parameter $d$ for driving angles (a) $\theta=45^{\circ}$ and (b) $\theta=135^{\circ}$. The coupling $\epsilon$ between the leads and the system is indicated in the inset of the figures. In (a) we also report the theoretical result (symbols) of Eq. (13). Other parameters are $k_{B} T=0.8$ and $\omega_{0}=3$ (in units of coupling strength).

pling constant $w_{L}=w_{R}=\epsilon$. We get the following expression for the total radiative (time-averaged) thermal energy flux $\overline{\mathcal{I}}$ per pumping area (see Appendix [42])

$$
\left.\tilde{\mathcal{I}} \approx \frac{\Omega}{2 \pi} \frac{\partial f}{\partial k}\right|_{\omega=\omega_{\mathrm{DP}}} \frac{4 \sqrt{3} \varepsilon^{4}\left(\varepsilon^{2}-\sqrt{2} d\right)}{\left[\left(\varepsilon^{2}-\sqrt{2} d\right)^{2}+12 \varepsilon^{4}\right]^{2}} .
$$

In Fig. 2a we report Eq. (13), together with the numerical calculations using Eq. $(4,6)$. We have performed similar calculations for different angles $\theta$ in order to validate the general features of Eq. (13). Specifically, in Fig. $2 b$, we report the behavior of $\overline{\mathcal{I}}$ versus the control parameter $d$ for $\theta=135^{0}$. In both cases we have found that $\overline{\mathcal{I}}$ diminishes for large values of the control parameter $d$ (see also Eq. (13)). In this limit, the Hamiltonian $H_{0}$ of the isolated system does not support a DP. As $|d| \rightarrow 0$, the DP is re-established, leading to an enhanced radiative thermal energy flux. This enhancement can be further boosted by decreasing the coupling $\epsilon$ between the system and the leads. As discussed previously, the coupling shifts the degenerate levels to the complex plane, thus lifting the DP degeneracy and therefore deteriorating the performance of the pump. We point out that typically, the extrema of $\overline{\mathcal{I}}$ is in the vicinity of the DP corresponding to $H_{0}(d=0 ; r=0)$ (see other cases at the Appendix [42]). This is due to the reminiscent effects of the coupling, which results in differences between $H_{0}$ and $H_{\text {eff }}$ (see Eq. (1)). Nevertheless, there can be appropriate choices of the control parameters $d, \epsilon$ for which these reminiscent effects disappears completely and the extrema of $\overline{\mathcal{I}}$ occurs at $d=0$, see Fig. $2 \mathrm{~b}$.

Adiabatic pumping using Circuits- An experimental demonstration of the effects of DPs on the adiabatically pumped thermal radiation, can be achieved using the electrical circuit shown in Fig. 1b. The system consists of a chain of three LC resonators with resonant frequency $\omega_{0}=1 / \sqrt{L C}$. The resonators are capacitively coupled along the chain via capacitances $\lambda C$. Moreover, the first and last resonators are coupled through a mutual inductance $M=\mu L$, leading to system coupling similar to Eq. 8. The circuit is connected in series to two terminal capacitors $C_{e}=\epsilon C$ which we initially consider as grounded, i.e. $V_{L(R)}=0$. For concreteness, we will set $\omega_{0} / 2 \pi=1 G H z, \lambda=0.2, \epsilon=0.1$, and $z_{0}=\omega_{0} L=1 / \omega_{0} C=70 \mathrm{Ohm}$. When $\mu=\mu_{\mathrm{DP}} \approx 0.13$, the eigen-frequencies of the grounded system demonstrate a DP degeneracy (see Supplement [42]).

We now turn the system into a scattering set-up by coupling each terminal capacitor $C_{e}$ to independent reservoirs at the same temperature $T$. These reservoirs are represented by a model for bandwidth limited Thevenin equivalent TEM transmission lines with characteristic impedance $Z_{0}=50 \mathrm{Ohms}$. The noise sources $V_{n}$ are synthesized such that $\left\langle V_{n}(\omega) V_{m}^{*}\left(\omega^{\prime}\right)\right\rangle=\frac{2 Z_{0}}{\pi} \Phi(\omega) \delta(\omega-$ $\left.\omega^{\prime}\right) \delta_{n m}$ where $\Phi(\omega)=k_{B} T \Theta(\omega)$. For demonstration purposes, we set $\Theta(\omega)=\sqrt{1-\left(\frac{\omega}{2 \omega_{c}}\right)^{2}}$, with $\omega_{c} \approx 0.47 \mathrm{GHz}$.

The pumping scheme is chosen to always enclose the DP when $\mu=\mu_{D P}$. Specifically, we consider a periodic modulation of the capacitances at left and right resonators such that $C_{1}(t)=C(1+r \sin (\Omega t)), \quad C_{2}(t)=$ $C(1+r \cos (\Omega t))$.

Next we inject into the circuit uncorrelated incoming waves of the same frequency $\omega$ and power $P_{s}=V_{s}^{2} /\left(8 Z_{0}\right)$ from the left $(\mathrm{L})$ and right $(\mathrm{R})$ reservoir. The average (over a cycle) net power flowing through the node $L(R)$ is obtained from the voltage $v_{L(R)}(t)$ and current $i_{L(R)}(t)$ sampled at the respective node $L(R)$ (see Supplement [42]). Specifically, $Q(\omega)$ is evaluated using Eq. (4) where the time-dependent energy current is $\mathcal{I}_{L(R)}(t, \omega)=v_{L(R)}(t, \omega) i_{L(R)}(t, \omega) / P_{s}$. In our simulations, we made sure the system reached a stationary state before the evaluation. The results from the time domain simulations are shown in Fig. 3a together with the outcome from the instantaneous $S^{t}$-matrix approach, see Eq. (6).[43] In the latter case we have extracted the instantaneous reflectance $R^{t}$ and reflection phase $\alpha^{t}$ using a standard scattering approach (see Supplement [42]) [44].

Having at our disposal the total radiative energy density $Q(\omega)$ for the circuit set-up, we are now able to incorporate $\Phi(\omega)$ for the total radiative thermal energy flux (per pumping area) passing through the system, $\overline{\mathcal{I}}$ versus $\mu$. In Fig. 3b we report our findings using the instantaneous $S^{t}$ matrix and the direct time-domain approaches. The data nicely demonstrates the enhancement in $\mathcal{I}$ due to the presence of the diabolic point as expected from the predictions of CMT.

Conclusions - We have introduced the concept of adiabatic thermal radiation pumps as a means to manage the direction of net radiative energy current for bodies in equilibrium. We addressed this problem by appropriately adopting, and establishing in the framework of resonant near-field thermal radiation, an instantaneous scattering matrix formalism borrowed from mesoscopic condensed matter. Using this tool, we highlighted the importance 

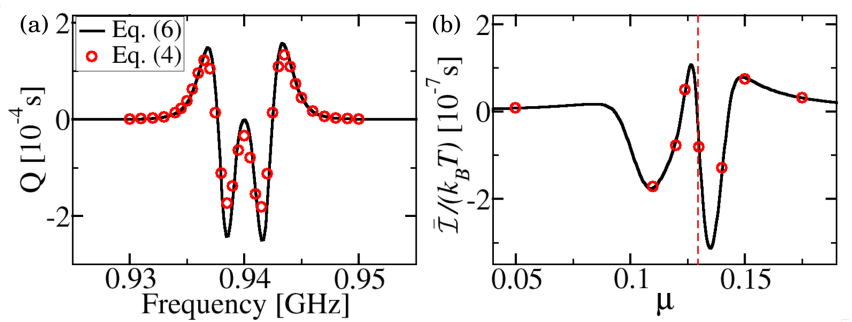

FIG. 3: (a) Total pumped energy density $Q(\omega)$ versus frequency for a typical value of the rescaled mutual inductance coupling at DP, $\mu=\mu_{\mathrm{DP}}$. The line is the numerical evaluation of $Q(\omega)$ using Eq. (6). The symbols are results from a direct time-domain simulation of net power at the left terminal. [43] (b) Total (averaged) pumped radiative energy current $\overline{\mathcal{I}}$ versus the mutual inductance $\mu$. The highest values of $\overline{\mathcal{I}}$ are reached at the proximity of the DP (red dashed line).

of wave interference effects in the field of thermal photonics for thermal radiation management. As an example, we demonstrated the impact of diabolic point spectral singularities in such framework. Our results have been tested against realistic simulations using electronic circuits, with the techniques directly applicable to systems where temporal control of resonant coupling is possible. An exciting application of our proposal might involve tunable superconducting resonators [45, 46] which will enable new forms of superconducting Q-bit manipulation. A future interesting direction is the study of the full counting statistics for thermal radiation. It will also be interesting to extend this study to other types of spectral singularities, like exceptional points [47]. These questions will be addressed in a subsequent publication.

Acknowledgements -(HL, LJFA, TK) acknowledge partial support by an ONR Grant No. N00014-16-1-2803, by a DARPA NLM program via Grant No. HR00111820042, by an AFOSR Grant No. FA 9550-14-1-0037, and by a NSF Grant No. EFMA-1641109. (BS) acknowledges the hospitality of Wesleyan Univ. where this work has been performed.

[1] A. Volokitin and B. Persson, Near-field radiative heat transfer and noncontact friction, Rev. Mod. Phys. 79, 1291 (2007).

[2] J. R. Howell, R. Siegel, M. P. Mengüs, Thermal Radiation Heat Transfer, (CRC, Boco Raton, FL), 5th Ed.

[3] T. L. Bergman, A. S. Lavine, F. P. Incropera, D. P. Dewitt, Introduction to Heat Transfer, (Willey, Hoboken, NJ), 6th Ed.

[4] S. Fan, Thermal Photonics and Energy Applications, Joule 1, 264 (2017).

[5] D. A. B. Miller, L. Zhu, S. Fan, Universal modal radiation laws for all thermal emitters, PNAS 114, 4336 (2017).

[6] L. Zhu, S. Fan, Near-complete violation of detailed balance in thermal radiation, Phys. Rev. B 90, 220301
(2014).

[7] W. C. Snyder, Z. Wan, X. Li, Thermodynamic constraints on reflectance reciprocity and Kirchoff's law, Appl. Opt. 37, 3464 (1998)

[8] Y. Hadad, J. C. Soric, A. Alú, Breaking temporal symmetries for emission and absorption, PNAS 113, 3471 (2016)

[9] A. W. Rodriguez, O. Ilic, P. Bermel, I. Celanovic, J. D. Joannopoulos, M. Soljacic, S. G. Johnson, FrequencySelective Near-Field Radiative Heat Transfer between Photonic Crystal Slabs: A Computational Approach for Arbitrary Geometries and Materials, Phys. Rev. Lett. 107, 114302 (2011).

[10] P. Ben-Abdallah and S.-A. Biehs, Near-Field Thermal Transistor, Phys. Rev. Lett. 112, 044301 (2014).

[11] S. Shen, A. Narayanaswamy, G. Chen, Surface Phonon Polaritons Mediated Energy Transfer between Nanoscale Gaps, Nano Lett. 9, 2909 (2009).

[12] E. Rousseau, A. Siria, G. Jourdan, S. Volz, F. Comin, J. Chevrier, and J.-J. Greffet, Radiative heat transfer at the nanoscale, Nat. Photonics 3, 514 (2009).

[13] R. S. Ottens, V. Quetschke, S. Wise, A. A. Alemi, R. Lundock, G. Mueller, D. H. Reitze, D. B. Tanner, B. F. Whiting, Near-Field Radiative Heat Transfer between Macroscopic Planar Surfaces, Phys. Rev. Lett. 107, 014301 (2011).

[14] T. Kralik, P. Hanzelka, M. Zobac, V. Musilova, T. Fort, and M. Horak, Strong Near-Field Enhancement of Radiative Heat Transfer between Metallic Surfaces, Phys. Rev. Lett. 109, 224302 (2012).

[15] R. St-Gelais, B. Guha, L. Zhu, S. Fan, and M. Lipson, Demonstration of Strong Near-Field Radiative Heat Transfer between Integrated Nanostructures, Nano Lett. 14, 6971 (2014).

[16] J. Shi, B. Liu, P. Li, L. Y. Ng, S. Shen, Near-Field Energy Extraction with Hyperbolic Metamaterials, Nano Lett. 15, 1217 (2015).

[17] K. Kim, B. Song, V. Fernandez-Hurtado, W. Lee, W. Jeong, L. Cui, D. Thompson, J. Feist, M. T. H. Reid, F. J. Garciá-Vidal, J. C. Cuevas, E. Meyhofer, and P. Reddy, Radiative heat transfer in the extreme near field, Nature 528, 387 (2015).

[18] B. Song, Y. Ganjeh, S. Sadat, D. Thompson, A. Fiorino, V. Fernandez-Hurtado, J. Feist, F. J. Garcia-Vidal, J. C. Cuevas, P. Reddy, E. Meyhofer, Enhancement of nearfield radiative heat transfer using polar dielectric thin films, Nat. Nanotechnol. 10, 253 (2015).

[19] R. St-Gelais, L. Zhu, S. Fan, and M. Lipson, Near-field radiative heat transfer between parallel structures in the deep subwavelength regime, Nat. Nanotechnol. 11, 515 (2016).

[20] B. Song, D. Thompson, A. Fiorino, Y. Ganjeh, P. Reddy, and E. Meyhofer, Radiative heat conductances between dielectric and metallic parallel plates with nanoscale gaps, Nat. Nanotechnol. 11, 509 (2016).

[21] S. Y. Lin, J. Moreno, and J. G. Fleming, Threedimensional photonic-crystal emitter for thermal photovoltaic power generation, Appl. Phys. Lett. 83, 380 (2003).

[22] A. Narayanaswamy, G. Chen, Surface modes for near field thermophotovoltaics Appl. Phys. Lett. 82, 3544 (2003).

[23] S. Basu, Z. M. Zhang, and C. J. Fu, Review of near-field thermal radiation and its application to energy conversion 
Int. J. Energy Res. 33, 1203 (2009).

[24] O. Ilic, M. Jablan, J. D. Joannopoulos, I. Celanovic, M. Soljacic, Overcoming the black body limit in plasmonic and graphene near-field thermophotovoltaic systems, Opt. Express 20, A366 (2012).

[25] Y. De Wilde, F. Formanek, R. Carminati, B. Gralak, P-A Lemoine, K. Joulain, J-P Mulet, Y. Chen, J-J Greffet, Thermal radiation scanning tunnelling microscopy, Nature 444, 740 (2006).

[26] A. Kittel, W. Müller-Hirsch, J. Parisi, S-A Biehs, D. Reddig, M Holthaus, Near-Field Heat Transfer in a Scanning Thermal Microscope, Phys. Rev. Lett. 95, 224301 (2005)

[27] C. R. Otey, W. Tung Lau, S. Fan, Thermal Rectification through Vacuum, Phys. Rev. Lett. 104, 154301 (2010)

[28] S. Basu, M. Francoeur, Near-field radiative transfer based thermal rectification using doped silicon, Appl. Phys. Lett. 98, 113106 (2011).

[29] L. Zhu, C. R. Otey, S. Fan, Negative differential thermal conductance through vacuum, Appl. Phys. Lett. 100, 044104 (2012).

[30] B. Guha, C. Otey, C. B. Poitras, S. Fan, and M. Lipson, Near-Field Radiative Cooling of Nanostructures, Nano Lett. 12, 4546 (2012).

[31] P. W. Brouwer, Scattering approach to parametric pumping, Phys. Rev. B 58, R10135 (1998).

[32] D. J. Thouless, Quantization of particle transport, Phys. Rev. B 27, 6083 (1983).

[33] B. L. Altshuler and L. I. Glazman, Pumping Electrons, Science 283, 1864 (1999).

[34] O. Entin-Wohlman, A. Aharony, and Y. Levinson, Adiabatic transport in nanostructures, Phys. Rev. B 65, 195411 (2002).

[35] J. E. Avron, A. Elgart, G. M. Graf, and L. Sadun, Optimal Quantum Pumps, Phys. Rev. Lett. 87, 236601 (2001).

[36] D. Cohen, Quantum pumping and dissipation: From closed to open systems, Phys. Rev. B 68, 201303(R) (2003).
[37] M. Switkes, C. M. Marcus, K. Campman, and A. C. Gossard, An Adiabatic Quantum Electron Pump, Science 283, 1905 (1999).

[38] L. Zhu, S. Sandhu, C. Otey, S. Fan, M. B. Sinclair, T. S. Luk, Temporal coupled mode theory for thermal emission from a single thermal emitter supporting either a single mode or an orthogonal set of modes, Appl. Phys. Lett. 102, 103104 (2013).

[39] A. Karalis, J. D. Joannopoulos, Temporal coupled-mode theory model for resonant near-field thermophotovoltaics, Appl. Phys. Lett. 107, 141108 (2015)

[40] H. Li, S. Suwunnarat, R. Fleischmann, H. Schanz, and T. Kottos, Random Matrix Theory Approach to Chaotic Coherent Perfect Absorbers, Phys. Rev. Lett. 118, 044101 (2017).

[41] Note that $Q(\omega)$ does not depend on the position $x_{0}$ where the flux is evaluated because on average no net energy is accumulated in the set-up.

[42] See Supplement for further details.

[43] Circuit simulations were performed using NGSpice V. 28plus, http://ngspice.sourceforge.net/

[44] Z. Lin, J. Schindler, F. Ellis, T. Kottos, Experimental observation of the dual behavior of PT-symmetric scattering, Phys. Rev. A 85, 050101 (2012).

[45] A. A. Adamyan, S. E. Kubatkin, A. V. Danilov, Tunable superconducting microstrip resonators, Applied Physics Letters 108, 172601 (2016).

[46] C. Bockstiegel, Y. Wang, M. R. Vissers, L. F. Wei, Chaudhuri, Hubmayr, J. Gao, A tunable coupler for superconducting microwave resonators using a nonlinear kinetic inductance transmission line, Applied Physics Letters 108, 222604 (2016).

[47] M. Chitsazi, H. Li, F. M. Ellis, T. Kottos, Experimental Realization of Floquet PT-Symmetric Systems, Phys. Rev. Lett. 119, 093901 (2017). 


\title{
Adiabatic Thermal Radiation Pumps for Thermal Photonics
}

\author{
Huanan $\mathrm{Li}^{1,2 *}$, Lucas J. Fernández-Alcázar ${ }^{1 *}$, Fred Ellis ${ }^{1}$, Boris Shapiro ${ }^{3}$, Tsampikos Kottos $^{1}$ \\ ${ }^{1}$ Wave Transport in Complex Systems Lab, Department of Physics, \\ Wesleyan University, Middletown, CT-06459, USA \\ ${ }^{2}$ Photonics Initiative, Advanced Science Research Center, CUNY, NY 10031, USA \\ ${ }^{3}$ Technion - Israel Institute of Technology, Technion City, Haifa 32000, Israel
}

(Dated: June 4, 2019)

\section{Supplemental Materials}

\section{DERIVATION OF EQ. (13)}

For "the examples of adiabatic pump" in the main text, we assume the driving angle $\theta=45^{\circ}$ and consider that the system is coupled with two identical tight-binding leads with equal coupling strength $\varepsilon$. The tight-binding leads supports a dispersion $\omega=\omega_{0}-2 \cos k$ in unit of coupling. In this case, the renormalization term $\Lambda$ appearing in Eq. (1) of the main text take the specific form $\Lambda=-\frac{1}{2} \cot (k) W W^{T}$ with the coupling matrix $W$ being

$$
W=\sqrt{2 \sin k}\left[\begin{array}{cc}
\varepsilon & 0 \\
0 & 0 \\
0 & \varepsilon
\end{array}\right] .
$$

Notice that the free space dispersion is a special case for which $k \approx \pi / 2$ (wide-band approximation) and thus $\Lambda=0$. On the other hand, the instantaneous effective Hamiltonian $H_{\mathrm{eff}}=H_{\mathrm{eff}}^{(0)}+\triangle^{t}$ reads

$$
H_{\mathrm{eff}}^{(0)}=\left[\begin{array}{ccc}
\omega_{0}+\varepsilon_{k}(d) & -1 & 1 \\
-1 & \omega_{0} & -1 \\
1 & -1 & \omega_{0}+\varepsilon_{k}(d)
\end{array}\right],
$$

and $\triangle^{t}=\operatorname{diag}\left(\delta v^{t}, 0, \delta u^{t}\right)$ where $\varepsilon_{k}(d)=-\varepsilon^{2} e^{\imath k}+\frac{d}{\sqrt{2}}, \delta v^{t}=r \sin \Omega t$ and $\delta u^{t}=r \cos \Omega t$. The eigenvalue system of $H_{\mathrm{eff}}^{(0)}=H_{\mathrm{eff}}^{(0), T}$, i.e., $\left.\left.H_{\mathrm{eff}}^{(0)} \mid a_{i}\right)=a_{i} \mid a_{i}\right), i=1,2,3$., can be given explicitly

$$
\begin{aligned}
& a_{1}=\omega_{0}-1+\varepsilon_{k}(d) \\
& a_{2}=\omega_{0}+\frac{1}{2}\left[1+\varepsilon_{k}(d)-\sqrt{9+\varepsilon_{k}(d)\left(2+\varepsilon_{k}(d)\right)}\right] \\
& a_{3}=\omega_{0}+\frac{1}{2}\left[1+\varepsilon_{k}(d)+\sqrt{9+\varepsilon_{k}(d)\left(2+\varepsilon_{k}(d)\right)}\right]
\end{aligned}
$$

and

$$
\begin{aligned}
& \left.\mid a_{1}\right)=\frac{1}{\sqrt{2}}\left[\begin{array}{lll}
-1 & 0 & 1
\end{array}\right]^{T} \\
& \left.\mid a_{2}\right)=\frac{1}{\sqrt{2+\left(a_{3}-\omega_{0}\right)^{2}}}\left[\begin{array}{lll}
1 & a_{3}-\omega_{0} & 1
\end{array}\right]^{T} \\
& \left.\mid a_{3}\right)=\frac{1}{\sqrt{2+\left(a_{2}-\omega_{0}\right)^{2}}}\left[\begin{array}{ccc}
1 & a_{2}-\omega_{0} & 1
\end{array}\right]^{T} \text {. }
\end{aligned}
$$

Due to the transposition symmetry of the matrix $H_{\mathrm{eff}}^{(0)},\left(a_{i}|\equiv| a_{i}\right)^{T}$ together with $\left.\mid a_{i}\right)$ constitute a complete biorthonormal basis. Correspondingly, using Eq. (9), we have the radiative energy density

$[*]$ These authors contributed equally to the results of this paper 


$$
Q(\omega)=\frac{\partial}{\partial \omega} P(k, d, \varepsilon)
$$

where

$$
P(k, d, \varepsilon)=\frac{\frac{d \delta \omega}{d k} \frac{\varepsilon^{6}\left(2-\varepsilon^{2}\right)}{\left(1-\varepsilon^{2}\right)^{4}}\left(4-\delta \omega^{2}\right) \delta \omega(\delta \omega+1)^{2}\left(\delta \omega-\omega_{5}\right)\left(\delta \omega-\omega_{6}\right)}{\left(\delta \omega-\omega_{1}\right)^{2}\left(\delta \omega-\omega_{1}^{*}\right)^{2}\left(\delta \omega-\omega_{2}\right)^{2}\left(\delta \omega-\omega_{2}^{*}\right)^{2}\left(\delta \omega-\omega_{3}\right)^{2}\left(\delta \omega-\omega_{4}\right)^{2}}
$$

with the frequency detuning $\delta \omega \equiv \omega-\omega_{0}=-2 \cos k$ and in the limit of $d \sim O\left(\varepsilon^{2}\right) \rightarrow 0$

$$
\begin{aligned}
& \omega_{1} \approx-1+\frac{\sqrt{2}}{2} d+\frac{1}{2}(-1+\imath \sqrt{3}) \varepsilon^{2} \\
& \omega_{2} \approx-1+\frac{\sqrt{2}}{6} d+\frac{1}{6}(-1+\imath \sqrt{3}) \varepsilon^{2} \\
& \omega_{3} \approx \omega_{4} \approx 2+\frac{\sqrt{2}}{3} d+\frac{2}{3} \varepsilon^{2} \\
& \omega_{5} \approx 1+\frac{\sqrt{2}}{4} d+\frac{1}{4} \varepsilon^{2} \\
& \omega_{6} \approx-1+\frac{\sqrt{2}}{4} d-\frac{1}{4} \varepsilon^{2} .
\end{aligned}
$$

For the thermal energy flux per pumping area $\overline{\mathcal{I}}$, Eq. (10) in the main text can be rewritten as

$$
\overline{\mathcal{I}}=-\frac{\Omega}{2 \pi} \int_{0}^{\pi} \frac{d k}{2 \pi} P(k, d, \varepsilon) \frac{\partial}{\partial k} f\left(\omega_{0}-2 \cos k\right) .
$$

The major contribution of $P(k, d, \varepsilon)$ to the integral mainly comes from $k$ values of short range around DP satisfying $\delta \omega \approx-1$, see Eq. (S6). Therefore we can simplify Eq. (S8) for $\overline{\mathcal{I}}$ to be

$$
\begin{aligned}
\overline{\mathcal{I}} & \approx-\left.\frac{\Omega}{2 \pi} \frac{\partial f}{\partial k}\right|_{\delta \omega=-1} \frac{\varepsilon^{6}\left(2-\varepsilon^{2}\right)}{\left(1-\varepsilon^{2}\right)^{4}} \frac{3\left(1+\omega_{5}\right)}{\left(1+\omega_{3}\right)^{2}\left(1+\omega_{4}\right)^{2}} I_{R} \\
I_{R} & \equiv \int_{-2}^{2} \frac{d(\delta \omega)}{2 \pi} \frac{(\delta \omega+1)^{2}\left(\delta \omega-\omega_{6}\right)}{\left(\delta \omega-\omega_{1}\right)^{2}\left(\delta \omega-\omega_{1}^{*}\right)^{2}\left(\delta \omega-\omega_{2}\right)^{2}\left(\delta \omega-\omega_{2}^{*}\right)^{2}} .
\end{aligned}
$$

After extending the integration range of $\delta \omega$ to the whole real axis and using residue theorem, we can evaluate $I_{R}$ in Eq. (S9) approximately. Finally, we have

$$
\left.\overline{\mathcal{I}} \approx \frac{\Omega}{2 \pi} \frac{\partial f}{\partial k}\right|_{\delta \omega=-1} \frac{4 \sqrt{3} \varepsilon^{4}\left(\varepsilon^{2}-\sqrt{2} d\right)}{\left[\left(\varepsilon^{2}-\sqrt{2} d\right)^{2}+12 \varepsilon^{4}\right]^{2}}
$$

Here, we also report the numerical calculations of $\overline{\mathcal{I}} \frac{2 \pi}{\Omega}$ versus $d$ for several different angles $\theta=0^{\circ}, 90^{\circ}$ in addition to what we show in the main text. As shown in Fig. S1, the extrema is also in the vicinity of the DP similar to the scenario $\theta=45^{\circ}$ shown in Fig. 2(a) of the main text.

\section{EIGENFREQUENCIES OF THE ELECTRICAL CIRCUIT}

Let us consider that capacitors $C_{e}$ are grounded, then $V_{L(R)}=0$. At steady state, voltages at nodes $i=1, c, 2$ (see Fig. 1b) are given by $v_{i}(t)=\operatorname{Re}\left(V_{i} e^{\imath \omega t}\right)$, where $\omega$ is the angular frequency. The Kirchoff equations for the voltage amplitudes $V_{1}, V_{c}$, and $V_{2}$ can be written in matrix form as $A \vec{V}=0$,

$$
\left(\begin{array}{ccc}
\omega^{2} \tilde{C}_{1}-1 / \tilde{L} & -\omega^{2} C \lambda & \mu / \tilde{L} \\
-\omega^{2} C \lambda & \omega^{2} \tilde{C}_{c}-1 / L & -\omega^{2} C \lambda \\
\mu / \tilde{L} & -\omega^{2} C \lambda & \omega^{2} \tilde{C}_{2}-1 / \tilde{L}
\end{array}\right)\left(\begin{array}{c}
V_{1} \\
V_{c} \\
V_{2}
\end{array}\right)=0
$$



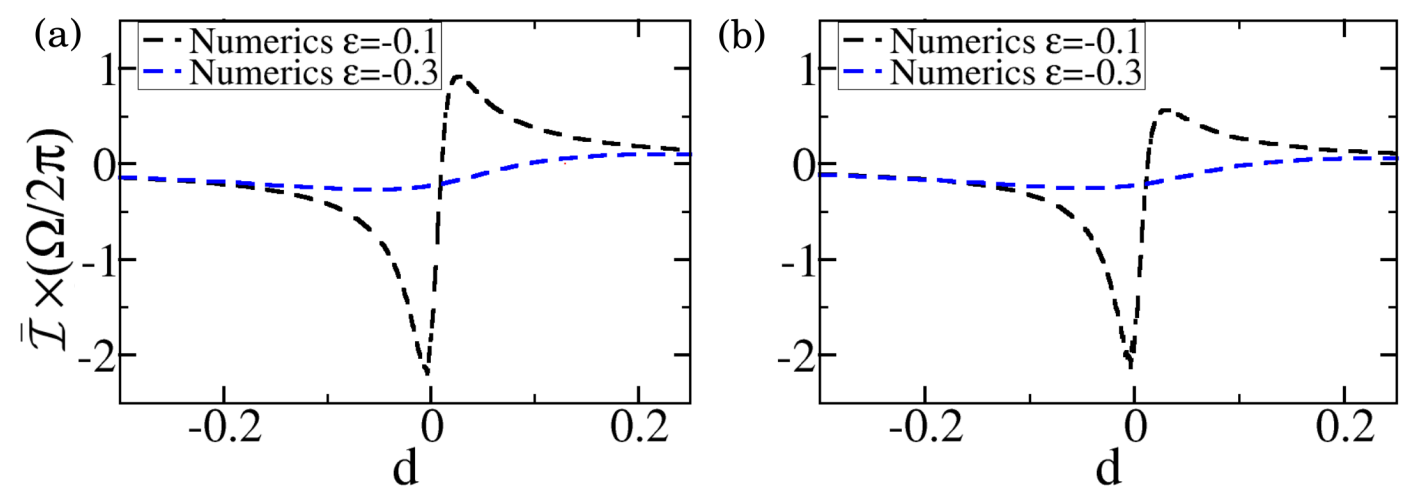

FIG. S1: Numerical calculation of $\overline{\mathcal{I}} \frac{2 \pi}{\Omega}$ versus $d$ for two different coupling strengths $\varepsilon=-0.1,-0.3$ when (a) $\theta=0^{\circ}$, (b) $\theta=100^{\circ}$. Other parameters are $\beta=0.8$ and $\omega_{0}=3$.

where $\tilde{C}_{1}=C_{e}+C_{1}+C \lambda, \tilde{C}_{c}=(C+2 C \lambda), \tilde{C}_{2}=C_{e}+C_{2}+C \lambda$, and $\frac{1}{\tilde{L}}=\frac{L}{L^{2}-\mu^{2} L^{2}}$.

The eigenfrequencies are those values of $\omega$ that give a determinant $\operatorname{det}(A)=0$. In absence of driving $C_{1}=C_{2}=C$, and using $\Omega=\left(\omega / \omega_{0}\right)^{2}, C_{e} / C=\epsilon$, the eigenfrequencies can be written as

$$
\begin{aligned}
\Omega_{1,2} & =\frac{2+\tilde{\epsilon}_{\mu}+\tilde{\mu}}{a} \pm \frac{\sqrt{\tilde{\mu}^{2}+2 \lambda\left[-\epsilon-\mu+\mu\left(\tilde{\epsilon}_{\mu}-\epsilon\right)\right]+\tilde{\epsilon}_{\mu}^{2}}}{a} \\
\Omega_{3} & =-\frac{1}{(\mu-1)(1+\lambda+\varepsilon)} .
\end{aligned}
$$

Here, $\tilde{\epsilon}_{\mu}=\epsilon+\mu(\epsilon+1), \tilde{\mu}=(3+\mu) \lambda$, and $a=2(1+\mu)[(2 \epsilon+3) \lambda+\epsilon+1]$. Given $\lambda$ and $\epsilon$, the solutions $\Omega_{1}$ and $\Omega_{3}$ take the same value at a particular $\mu=\mu_{D P}$. For the case $\lambda=0.2, \epsilon=0.1$, we have $\mu_{\mathrm{DP}} \approx 0.129506$. We note that this is not an avoided crossing, but a rare occurrence called a diabolic point.

\section{SCATTERING PARAMETERS FOR THE ELECTRICAL CIRCUIT}

Now, let us consider the system depicted in Fig. $1 \mathrm{~b}$ as a scatterer where the voltages on the left and right nodes, $V_{L}$ and $V_{R}$, are not grounded. Instead, they are driven by time dependent voltage sources $v_{L(R)}^{s}(t)=\operatorname{Re}\left(V_{L(R)}^{s} e^{\imath \omega t}\right)$. We can split both the voltages at, and the currents passing through, the nodes $L(R)$ from the model transmission line as a sum of two contributions,

$$
\begin{aligned}
v_{L(R)}(t, \omega) & =\operatorname{Re}\left[\left(V_{L(R)}^{+}+V_{L(R)}^{-}\right) e^{\imath \omega t}\right], \\
i_{L(R)}(t, \omega) & =\frac{1}{Z_{0}} \operatorname{Re}\left[\left(V_{L(R)}^{+}-V_{L(R)}^{-}\right) e^{\imath \omega t}\right] .
\end{aligned}
$$

Here $V_{L}^{+}=V_{L}^{s} / 2$ and $V_{R}^{-}=V_{R}^{s} / 2$ are the complex voltage amplitudes of the incoming waves from left and right Thevenin equivalent sources; and $V_{L}^{-}$and $V_{R}^{+}$are the voltage amplitudes of the outgoing scattered waves (see green arrows in Fig. 1b). In the particular case in which $V_{R}^{-}=0, V_{L}^{-}\left(V_{R}^{+}\right)$is the amplitude of the reflected (transmitted) wave. Then, $r_{L}=\frac{2 V_{L}^{-}}{V_{s}}\left(t_{R, L}=\frac{2 V_{R}^{+}}{V_{s}}\right)$ is the reflection (transmission) coefficient. Similarly, when $V_{L}^{+}=0$, the reflection (transmission) coefficient is $r_{R}=\frac{2 V_{R}^{+}}{V_{s}}\left(t_{L, R}=\frac{2 V_{L}^{-}}{V_{s}}\right)$. 